groups, respectively: 8 (4-31) vs. 1.5 (1-5) cells $/ \mathrm{mm}^{3} ; 87 \%$ (77-94) vs. $83 \%(68-92)$ lymphocytes and $8 \%(4-12)$ vs. $15 \%(6-$ 27) monocytes $/ \mathrm{mm}^{3}$; protein $47(35-69)$ vs. $35(30-38) \mathrm{mg} / \mathrm{dL}$; and glucose $52(43-63)$ vs $58(53-66) \mathrm{mg} / \mathrm{dL}$. $31 \%$ of patients had a reactive VDRL in CSF. HIV+ were shown to be of younger age $(p=0.003)$ and to present significantly higher CSF cell counts $(p=0.002)$ and protein $(p=0.008)$.

Conclusion In our cohort most patients were HIV+, who developed the disease at an earlier age. CSF parameters showed significantly different cell counts and protein concentrations among HIV+. CSF VDRL presented a low sensitivity in both groups, with no significant difference between them. $\mathrm{HIV}+$ patients were asymptomatic in more than half of cases

\section{P3.242 FACTORS ASSOCIATED WITH VAGINAL PROSTATE- SPECIFIC ANTIGEN DETECTION AND SELF-REPORTED UNPROTECTED SEX IN A CLINICAL TRIAL IN MALAWI}

\begin{abstract}
${ }^{1}$ Yasaman Zia, ${ }^{1}$ Nicole Davis, ${ }^{1}$ Jeffrey Wiener, ${ }^{2}$ Donna Lapple, ${ }^{3}$ Lameck Chinula, ${ }^{3}$ Jennifer Tang, ${ }^{3}$ Gerald Tegha, ${ }^{3}$ Albans Msika, ${ }^{2}$ Marcia Hobbs, ${ }^{1}$ Athena Kourtis. ${ }^{1}$ Centres for Disease Control (CDC), Atlanta, GA, USA; ${ }^{2}$ University of North Carolina (UNC), Chapel Hill, NC, USA; ${ }^{3}$ UNC-Project Malawi, Lilongwe - Malawi
\end{abstract}

\subsection{6/sextrans-2017-053264.477}

Introduction Vaginal prostate-specific antigen (PSA) is a biomarker of recent semen exposure that may overcome potential fallacies of self-reported sexual behaviours, improving sexual exposure assessment in HIV/STI research.

Methods We conducted a cohort study to compare PSA detection and self-reported unprotected sex using data from a clinical trial in Malawi that randomised 73 HIV-infected and 24 HIV-uninfected women to depot medroxyprogesterone acetate injectable or levonorgestrel implant. Women were advised to abstain from vaginal intercourse or use a condom the day preceding study visits. We tested 539 vaginal swabs from 97 women for PSA using the ABAcard p30 rapid strip test (Abacus Diagnostics, Inc., West Hills, CA). Self-reported sexual behaviours and vaginal swabs were collected at six study visits. Log-binomial regression with generalised estimating equations were used to estimate associations of PSA detection and reported unprotected sex with demographic and behavioural factors, adjusting for study arm and pre- vs. post-contraception initiation.

Results Overall, 55 (57\%) women tested positive for PSA and 54 (56\%) women reported unprotected sex. Among PSA-positive samples, $62 \%(65 / 105)$ of instances reported no unprotected sex. The following were associated with PSA detection: HIV-negative status (Prevalence Ratio (PR): 1.69, 95\% Confidence Interval (CI):1.09, 2.61), younger age (PR: 1.04, CI: $1.00,1.08$ ), reported unprotected sex (PR: 2.48, CI: 1.70, 3.60 ), and sex within past 48 hours (PR: 4.68, CI: 3.00, 7.30). The same factors were significantly associated with selfreported unprotected sex, as was PSA detection (PR: 2.45, CI: $1.7,3.53)$.

Conclusions Self-reported sexual behaviours were significantly associated with PSA detection. However, PSA was detected among women reporting no unprotected sex, suggesting misreporting of condom use or condom failure. HIV-negative status and younger age were associated with unprotected sex, suggesting more frequent biases in research data for these groups based on social desirability and non-adherence to protocols.

\section{P3.243 NEISSERIA GONORRHOEAE ANTIMICROBIAL SUSCEPTIBILITIES SURVEILLANCE IN CHINA}

Yueping Yin, Xiuqin Dai, Shaochun Chen, Yan Han, Xianfsheng Chen. National Centre For Std Control, China Cdc, Nanjing - China Popular Republic

\subsection{6/sextrans-2017-053264.478}

Introduction Gonococcal infections have been a major sexually transmitted disease (STD) since 1980s'and also a serious public health problem in China. A national surveillance report showed that the number of reported gonorrhoea cases were 100245 in 2015. Resistant gonococcal infections are the obstacle in control work of the disease, which not only affect the treatment as well as the development of complications, but also facilitate the further transmission. The WHO has been drafted a global action plan to control the spread and impact of antimicrobial resistance in Neisseria Gonorrhoeae. This report will to analysis the susceptibility trends of NG to ciprofloxacin, spectinomycin, and ceftriaxone from 2005 to 2013 in China including PPNG and TRNG rate.

Methods The production of $\beta$-lactamase was determined by paper acidometric testing method. Antimicrobial susceptibility testing was performed for tetracycline, ciprofloxacin, spectinomycin, and ceftriaxone using the agar dilution method. Establishment of quality control standards and system, which provide regular examination of participant laboratories through sending quality control strains, as well as through field supervision in China.

Results About 11625 NG isolates were collected from patients with uncomplicated gonorrhoea in 2005-2013. A total of 4565 (39.27\%) PPNG were and 4807 (41.21\%) TRNG were identified, with an increase from $28.09 \%$ in 2005 to $39.15 \%$ in 2013. A total of 11178 (96.16\%) ciprofloxacin-resistant strains were identified and the rate of ciprofloxacin-resistant strains keep stable from 2005 to 2013. All of isolates were susceptible to spectinomycin and ceftriaxone from 2005-2013, but a total of 4325 (36.79) ceftriaxone-intermediate strains were identified.

Conclusion Antimicrobial susceptibility of NG isolated in China from 2005 to 2013 was characterised by high rate of PPNG, TRNG, and ciprofloxacin -resistant strains. Results of the study illustrate that spectinomycin and ceftriaxone can be considered as the choice drugs for empirical treatment of infection in China.

\section{P3.244 GENETIC CHARACTERISTICS AND MOLECULAR MECHANISMS OF RESISTANCE OF AZITHROMYCIN- RESISTANT NEISSERIA GONORRHOEAE ISOLATED IN SHENZHEN, CHINA DURING 2011-2015}

${ }^{1}$ Zhang Lijun, ${ }^{2}$ Wang Feng, ${ }^{2}$ Peng Yi, ${ }^{2}$ Mo Junluan. ${ }^{1}$ Shenzhen Family Planning Service Centre, Shenzhen - China Popular Republic, ${ }^{2}$ Shenzhen Centre for Chronic Disease Control, Shenzhen - China Popular Republic

\subsection{6/sextrans-2017-053264.479}

Introduction Most current guidelines now recommend Azithromycin (AZM) as a component of dual therapy for gonorrhoea in combination with third-generation cephalosporins. Here we investigated the prevalence, molecular mechanisms and genetic characteristics of azithromycin-resistant(AZM-R) N.gonorrhoeae isolates in Shenzhen area from 2011 to 2015.

Methods A total of $788 \mathrm{~N}$. gonorrhoeae isolates were collected.The agar dilution method and E-test were used to 
determine the minimum inhibitory concentration (MIC) of these strains to AZM. All AZM-R isolates(MIC $\geq 2 \mu \mathrm{g} / \mathrm{ml}$ ) were screened for mutations in $23 \mathrm{~S}$ rRNA, $m$ trR and erm genes and genotyped using $N$. gonorrhoeae multi-antigen sequence typing (NG-MAST).

Results 50 (6.3\%) AZM-R isolates were collected, including 21 with azithromycin high-level resistant (AZM-HLR, MIC $\geq 256 \mu \mathrm{g} / \mathrm{ml}$ ) and 29 with azithromycin middle-level resistant (AZM-MLR, MIC between 2 to $16 \mu \mathrm{g} / \mathrm{ml}$ ). There were 18 strains had mutations (A2143G) and 12 strains had mutations (C2611T) in the four copies of the 23S rRNA gene in the AZ-HLR and AZ-MLR group respectively. Analysis of the promoter and coding region of $m t r R$ gene, significantly more AZM-HLR exhibited one pattern contained G45D and Y105H mutations compared with the AZM-MLR and AZ-S. Only one strains with positive PCR detection of ermB gene $(\mathrm{MIC}=2 \mu \mathrm{g} / \mathrm{ml})$. Among all the isolates, 81 sequence types (STs) were identified by NG-MAST, of which 8 STs were more often in the AZ-R group. Some STs (ST3356 and ST1866) that were observed had been noted in a previous reports of emerging AZM-R N. gonorrhoeae in Nanjing, Chongqing and Guangzhou. No resistant strains cluster was observed by NJ phylogenetic tree.

Conclusion AZM should not be recommended as a monotherapy for gonococcal in Shenzhen. The high-level and middlelevel resistance to AZM in $N$. gonorrhoeae were mainly mediated by specific mutations A2059G and C2611T in 23S rRNA respectively. Repeated emergence of ST1866 and ST3356 may help us to better monitor and analyse the epidemiological characteristics of AZM-R N. gonorrhoeae strains.

Support: Shenzhen Centre For Chronic Disease Control has been responsible for antimicrobial resistance surveillance program of N. gonorrhoeae in Shenzhen since 1992.

\section{LB3.245 GENOTYPING OF HIGH-RISK HUMAN PAPILLOMAVIRUS TYPES ASSOCIATED WITH PREDICTOR FACTORS TO CERVICAL CANCER IN WOMEN WITHOUT CYTOLOGICAL ABNORMALITIES: A CROSS SECTIONAL EPIDEMIOLOGICAL STUDY}

${ }^{1}$ Simões RSQ, ${ }^{2}$ Silva EP, ${ }^{1}$ Barth OM. 'Laboratory of Viral Morphology and Morphogenesis, Instituto Oswaldo Cruz, Fundação Oswaldo Cruz, Rio de Janeiro, RJ, Brazil, ${ }^{2}$ Vale do Itajaí University, Itajaí, Santa Catarina, Brazil

\subsection{6/sextrans-2017-053264.480}

Introduction The mucosal Human Papillomavirus (HPV) types have been described in the Alphapapillomavirus genus and infect the anogenital epithelium. There are viral groups based on their oncogenic activity as high-risk types, low-risk types and types of undetermined-risk. The aim of the study was to evaluate HPV frequency and co-factors related to cervical cancer in sexually active women from Rio de Janeiro city. This cross-sectional design study was performed at public health institutions from 2014 to 2016.

Methods Clinical samples were collected with a cervical cytobrush and stored at $-20^{\circ} \mathrm{C}$. After nucleic acid amplification, specific primers of high-risk types (HPV-16,-18,-31/-45) were used. Statistical analysis was applied in 18 socio-demographic variables.

Results This study demonstrated that of the 100 participants, $20 \%$ were DNA HPV positive. Of these, $8 \%$ were amplified by HPV-18/-45; and $12 \%$ were undetermined types. HPV-16 and -31 types were not detected. Comparing the genotypes,
$37.5 \%$ were predominately over 45 years old, $62.5 \%$ of respondents were HPV-18 positive, married and/or of co-habitation matrimonial status and62.5\% don't smoke. All of them don't use drugs and alcohol, four patients (50\%) have monthly income between one to four minimum wages, $37.5 \%$ started sexual activity before the age of 17 years and $50 \%$ had less than five sexual partners. Twenty five percent of the patients have had multiple infections and were unmarried. The 3\% who have had the both types of HPV infection had more than five sexual partners. A highly significant factor associated with HPV infection was who did not use the condom and had an income between one to four minimum wages, both with $87.5 \%$.

Conclusion This epidemiological study showed a strongly association among the presence of DNA HPV types with risk factors in unimmunized women. A large challenge to public health is cancer prevention in populations from developing countries with high risk exposure associated with the history of other sexually transmitted diseases (STDs) in different geographic regions.

Support: Coordenação de Aperfeiçoamento de Pessoal de Nivel Superior (CAPES)/Programa Brasil Sem Miséria; Conselho Nacional de Desenvolvimento Científico e Tecnológico (CNPq)

\section{LB3.246 PREVALENCE AND RISK FACTORS OF HUMAN PAPILLOMAVIRUS INFECTION IN UNIMMUNIZED WOMEN IN BRAZIL}

${ }^{1}$ Simões, RSQ, ${ }^{2}$ Silva EP, 'Barth OM. ' ${ }^{1}$ Laboratory of Viral Morphology and Morphogenesis, Instituto Oswaldo Cruz, Fundação Oswaldo Cruz, Rio de Janeiro, RJ, Brazil; ${ }^{2}$ Vale do Itajaí University, Itajaí, Santa Catarina, Brazil

10.1136/sextrans-2017-053264.481

Introduction The Brazilian Immunisation Program recommended the vaccine to be administered at 9 to 11 years of age before most adolescents became sexually active.The aim of this study is to describe the human papillomavirus(HPV) infection prevalence among unimmunized women and to identify the risk factors as demographic, behavioural and biological variables.

Methods An epidemiological study was performed in women randomly selected during 2014 to 2016. About 100 cervical cell scrapings were collected with a cytobrush. DNA extraction and HPV detection were performed by PCR, Nested PCR and specific primers. DNA quality was amplified by $\beta$-globin PCR primers. Restriction fragment length polymorphism (RFLP) patterns of L1 PCR products were used for genotyping.

Results In the population studied, HPV prevalence was of $20 \%$. HPV 18 and 45 were the most frequently detected HPV types. A group of 100 women was divided into six aged groups ( $\leq 25$ y.o; 26 to 30 y.o; 31 to 35 y.o; 36 to 40 y.o; 41 to 45 y.o; $\geq 45$ y.o).Distribution among racial/ethnic groups was representative when $47 \%$ of women were white and $53 \%$ were black and on the race/ethnicities. Most women were currently not married (56\%) and married or cohabitating (44\%) $(\mathrm{p}<0,05)$.Psychosocial and psychosexual issues demonstrated that six percent of the women exhibit history of sexually transmitted diseases, except HIV, with $85 \%$ of women having sex with until to five partners $(p<0,03)$. About the employment status of all the participating women, $90 \%$ reported having had at least one until four basic salaries. Only 5\% women have had higher education. The most women (87\%) have had secondary and fundamental education. 